\title{
Groundwater Assessment Platform (GAP): A new GIS tool for risk forecasting and mitigation of geogenic groundwater contamination
}

\author{
M. Berg \& J.E. Podgorski \\ Eawag, Swiss Federal Institute of Aquatic Science and Technology, Dübendorf, Switzerland
}

\begin{abstract}
Over 400 million people worldwide use groundwater contaminated with arsenic and/or fluoride as a source of drinking water. The Swiss Federal Institute of Aquatic Science and Technology (Eawag) has developed a method to estimate the risk of contamination in a given area using geological, topographical and other environmental data without having to test samples from every single well. The research group's knowledge is now being made available free of charge on the interactive Groundwater Assessment Platform (GAP, www.gapmaps.org). GAP is an online GIS platform for risk forecasting and mitigation of geogenic groundwater contamination. GAP enables researchers, authorities, NGOs and other professionals to visualize their own data and generate hazard risk maps for their areas of interest.
\end{abstract}

\section{INTRODUCTION}

A third of the world's population uses groundwater for drinking and cooking. Groundwater is generally a safe alternative to untreated, microbially contaminated surface water. However, about $10 \%$ of wells are contaminated with arsenic and/or fluoride.

Since health symptoms often only become visible after long-term exposure, it is important to identify safe and unsafe groundwater sources as early on as possible. In recent years, significant progress has been made in predictive risk modeling of arsenic contamination. Several studies identified a relatively small number of geological and hydrogeochemical parameters as significant spatial proxies that can be used to predict the regional distribution of high and low arsenic concentrations across entire regions, even in areas without survey data (Amini et al., 2008; Ayotte et al., 2017; Bretzler et al., 2017; Podgorski et al., 2017; Rodriguez-Lado et al., 2013; Winkel et al., 2008, 2011).

This approach has tremendous potential and provided the basis for Eawag to develop GAP for the mapping, sharing and predictive risk modeling of groundwater data.

\section{METHODS}

\subsection{Internet platform}

GAP was developed as an online open-source data and information-sharing portal for groundwater-related questions, with a special focus on geogenic contaminants (Fig. 1). It offers two main sections:

(1) GAP Maps where users can view existing data and risk maps, but most importantly use their own data to create risk maps (Fig. 2);

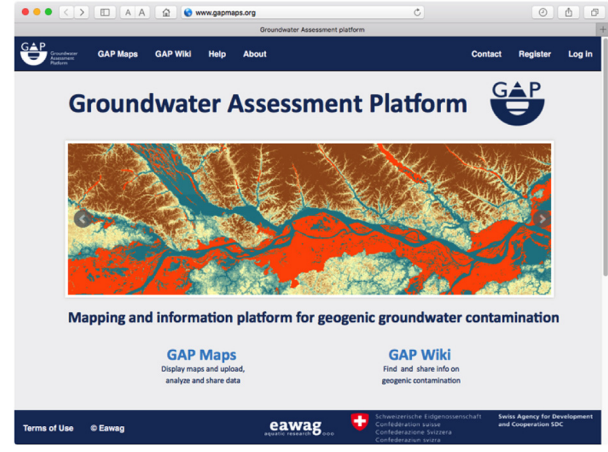

Figure 1. Entry page of the Groundwater Assessment Platform (www.gapmaps.org).

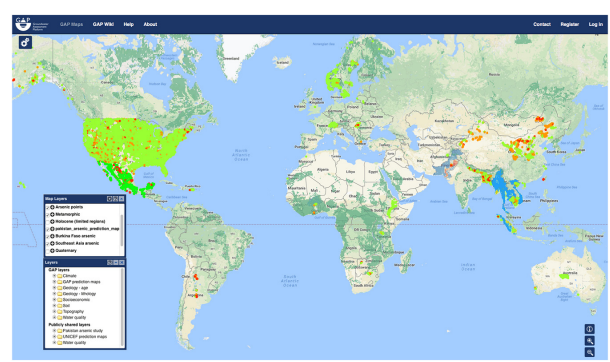

Figure 2. Existing arsenic data in GAP as of November 2017.

(2) GAP Wiki where users can view and contribute information on mitigation and issues surrounding geogenic contamination.

The high-level goals of GAP are aligned with the UN Sustainable Development Goal (SDG) 6, in particular 6.1 - Access to safe drinking water, 

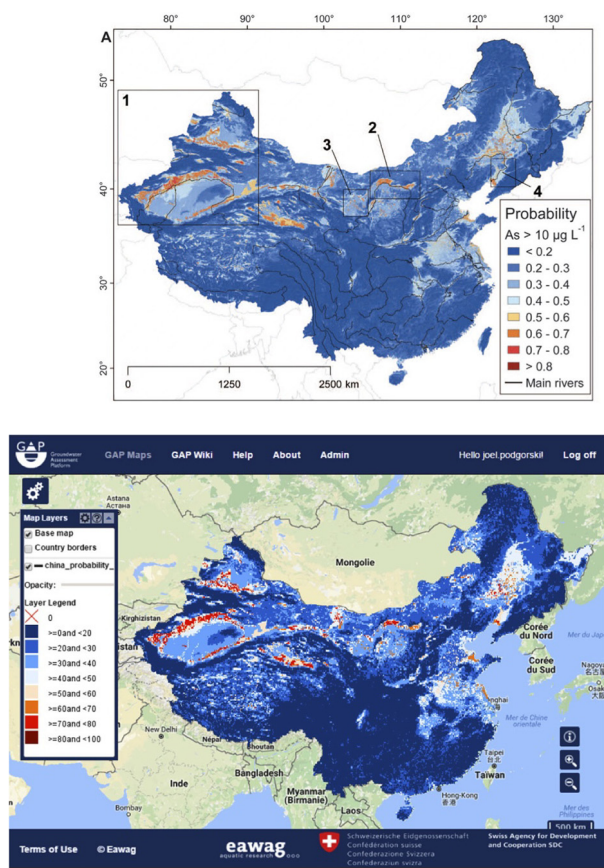

Figure 3. Arsenic prediction map for China modeled offline (Rodriguez-Lado et al., 2013) and the analog created in GAP (lower panel). The results between modeling via manual coding and modeling with GAP are obviously very similar.

6.2 - Pollution and hazardous chemicals and 6.B Community involvement in water management. Hence, GAP's mission is "To assist communities, national and international institutions, civil society and research organizations in having access to maps, data and relevant information to enable all people and the environment to have an equitable right to safe groundwater."

\section{RESULTS}

\subsection{Simple and fast creation of hazard prediction maps}

To showcase its modeling functionality, GAP was used to reproduce the arsenic hazard map of China that had been previously created (Rodríguez-Lado et al., 2013). The question was if the relatively basic features offered by GAP could achieve similar results and reproduce this published map, which was generated by a complex statistical modeling scheme that involved taking a weighted average of the best of 100 logistic regression iterations that used different random combinations of training and testing data. GAP indeed produced a very similar map as can be seen in Figure 3. Although some alternative datasets and a somewhat lower degree of sophistication (e.g. only one iteration and no random sampling) were necessary with GAP, the result below shows that a comparable model can be produced in GAP in a fraction of the time.

\section{OUTLOOK}

GAP enables users to visualize their own data with relatively little effort and to produce hazard maps with them. This makes it simple to identify wells that should be investigated as a matter of priority, such that available funds and resources can be deployed in a targeted manner.

This new platform facilitates rapid localization of geogenic contamination, which itself is a milestone in the protection of public health. But just as important as early detection is the development of practical methods for removing these toxic substances from water.

In spite of the progress made, a lot of research still needs to be done, especially on the development of locally adapted treatment technologies. Therefore, GAP was also designed with a wiki forum for sharing information. The sooner existing knowledge can be effectively disseminated, the better.

\section{ACKNOWLEDGEMENTS}

GAP is co-funded by the Swiss Agency for Development and Cooperation (SDC) and Eawag. We thank Manouchehr Amini, Ruth Arnheiter, Anja Bretzler, Jay Matta, Annette Johnson, Fabian Suter, Tobias Siegfried, Andreas Steiner, Jakob Steiner, and Chris Zurbrügg for their various contributions to the development of the GAP tool.

\section{REFERENCES}

Amini, M., Abbaspour, K.C., Berg, M., Winkel, L., Hug, S.J., Hoehn, E., Yang, H. \& Johnson, C.A. 2008. Statistical Modeling of global geogenic arsenic contamination in groundwater. Environ. Sci. Technol. 42: 3669-3675.

Ayotte, J.D., Medalie, L., Qi, S.L., Backer, L.C. \& Nolan, B.T. 2017. Estimating the high-arsenic domestic-well population in the conterminous United States. Environ. Sci. Technol. 51: 12443-12454.

Bretzler, A., Lalanne, F., Nikiema, J., Podgorski, J., Pfenninger, N., Berg, M. \& Schirmer, M. 2017. Groundwater arsenic contamination in Burkina Faso, West Africa: predicting and verifying regions at risk. Sci. Total Environ. 584-585, 958-970.

Podgorski, J.E., Eqani, S.A.M.A.S., Khanam, T., Ullah, R., Shen, H. \& Berg, M. 2017. Extensive arsenic contamination in high-pH unconfined aquifers in the Indus Valley. Science Advances 3, e1700935.

Rodriguez-Lado, L., Sun, G., Berg, M., Zhang, Q., Xue, H., Zheng, Q. \& Johnson, C.A. 2013. Groundwater arsenic contamination throughout China. Science 341: 866-868.

Winkel, L., Berg, M., Amini, M., Hug, S.J. \& Johnson, C.A. 2008. Predicting groundwater arsenic contamination in Southeast Asia from surface parameters. Nature Geosci. 1: $536-542$.

Winkel, L.H.E., Trang, P.T.K., Lan, V.M., Stengel, C., Amini, M., Ha, N.T., Viet, P.H. \& Berg, M. 2011. Arsenic pollution of groundwater in Vietnam exacerbated by deep aquifer exploitation for more than a century. Proc. Natl. Acad. Sci. U.S.A. 108: 1246-1251. 